\title{
Forecasting the New Trends about the Consumer Behavior in the Cruise Industry Post COVID-19
}

\author{
Ana Lucia Rodrigues da Silva, Reinaldo Castro Souza
}

The Research Group on Maritime and River Cruises, Pontifical Catholic University of Rio de Janeiro, PUC Rio, Metrology and Industrial Quality (Pos MQI), Rio de Janeiro, Brazil

Email: ana@sinerconsult.com.br, reinaldo@puc-rio.br

How to cite this paper: da Silva, A.L.R. and Souza, R.C. (2022) Forecasting the New Trends about the Consumer Behavior in the Cruise Industry Post COVID-19. Journal of Data Analysis and Information Processing, 10, 58-77.

https://doi.org/10.4236/jdaip.2022.101004

Received: January 8, 2022

Accepted: February 20, 2022

Published: February 23, 2022

Copyright $\odot 2022$ by author(s) and Scientific Research Publishing Inc. This work is licensed under the Creative Commons Attribution International License (CC BY 4.0).

http://creativecommons.org/licenses/by/4.0/

\begin{abstract}
This paper presented the new sanitary and health protocols implemented by cruise companies in order to internationally resuming the industry. The perception of Brazilians regarding these new protocols was identified through a quantitative survey with a sample of 412 Brazilian respondents, carried out between May and June 2021. As main results, sanitary and health protocols that do not affect their experiences on board were identified, as well as those protocols that compromised the experience perceived by the future traveler. The respondents' propensity to travel on cruise ships and their perceptions about the influence of the ship size, the number of ports of call, nationalities on board, the number of guests, among other aspects, were also analyzed. Finally, in the final section of this paper, we presented an estimation of the expected number of cruisers for the 21/22 cruising season in Brazil, based on a kind Bayesian argument, considering different scenarios for the forthcoming season.
\end{abstract}

\section{Keywords}

Cruise Industry, COVID-19, Sanitary and Health Protocols, Quantitative Survey, Forecasting, Brazilian's Cruisers

\section{Introduction}

The cruise industry is characterized as being an international, well-planned, innovative and capital-intensive industry. It has a huge supply chain and numerous crew members. It contributes economically to the cities and ports of call, including the embarkation and disembarkation ports and cities. The uninterrupted nature of its activities has always been one of its main characteristics, associated with its presence in all oceans and continents. 
The cruise industry, unlike other industries in the tourism and transport segment, has a long-term plan, which ensures high predictability and allows the sale of trips up to 3 years in advance, which has always been very well used by its frequent and equally well-planned customers. However, since March 2020, the entire cruise industry has stopped due to COVID-19 which remained until July 2021, when some cruise lines are going to resume with a part of their fleet.

The preparation to resume was collaborative work that had involved different stakeholders and specialists. As a result, there are a lot of new health and safety protocols in order to make a cruise trip as safe as possible.

The aim of this research was to verify how Brazilians feel about going on a cruise during this pandemic situation and their perception of the health and safety protocols adopted by cruise lines. Another goal of this paper is to forecast the next cruise season in Brazil, predicting the number of possible guests in different scenarios. For attaining this goal, this paper presents a Bayesian point prediction of the number expected of cruisers in Brazil season 21/22.

This paper presents a brief theory about experiential marketing, the background of the cruise industry, the new protocols onboard and ashore after COVID19 , the data from the quantitative research with 412 people, a forecasting of Brazilian cruise season 21/22, and a Bayesian point prediction of the number of cruisers in Brazil season 21/22. The conclusion and the applied questionnaire are also presented.

The experiential marketing theory was chosen to better understand the importance of well knowing the perception of cruise potential customers about resuming the cruise industry with a new routine and protocols on board and ashore.

Hirschman and Holbrook [1] published in 1982 the article "The Experiential Aspects of Consumption: Consumers Fantasies, Feelings and Fun". It was considered one of the first papers that claims the relevance of customer experience in customers' decision purchasing. The authors identified the necessity to examine the consumer's experience throughout the purchase process, in addition to placing customer emotion as a crucial aspect of consumption [2]. Hirschman and Holbrook, also cited by [3], emphasized the importance of organizations understanding and addressed more deeply about the emotional aspects of consumption, replacing with what was practiced until then, that is, explaining consumption phenomena only under a logical and certain point of view.

According to [1], the authors also defend that consumer decisions have their own criteria and motivations for decision making. It also depends not only on the characteristics of the product or service, but also on the aspects that satisfying their emotional desires. In this sense, the experiential view understands consumption as something fundamentally subjective. For Hirschman and Holbrook, its meaning and importance could vary from person to person, who will react differently to internal and external stimuli. The experience, according to the authors, is a "personal occurrence, often with an important emotional meaning, based on the interaction with the stimuli from the products or services consumed" [1]. 
According to Pontes [2], "the idea of offering the customer unique and valuable experiences has become a fundamental strategic focus among marketers". In this sense, experiential marketing seeks to create pleasant experiences not only at the time of purchase, but also at the moment of consumption and post-consumption, awakening the consumer's emotions, feelings and thoughts for better interaction with the product or service. Explain Moral Moral and Fernández Alles, cited by [3]: the central core of experience marketing is the experience in the course of consumption.

The cruise industry has many cruise lines (64 brands) and more than 250 ships. Its annual growth rate is 5.4\% [4], higher than the tourism sector itself, which is 4.9\% [5]. Its demand is composed by North American 52\%, Europe 26\%, the rest of the world 22\% of the total [4]. According to Cruise Lines International Association-CLIA [4], the main destinations for cruising are North America with $39 \%$ (the Caribbean is the most popular in this region), Europe with $28 \%$ (Mediterranean), Asia and Pacific with 14\% (Asia), and the rest of the world with 18\% (South America).

Before the COVID-19, this industry had experienced some trends, according to Dr. Baker, in "The Cruise Industry: Past, Present and Future", such as: increasing embarkations from close-to-home ports, a greater focus on family travel, exotic spots and itineraries, growing variety of ships, growing of cruises in a specific market, a boom in thematic cruises, focus on China, Asia and new destinations [6].

Due to COVID-19, the cruise ships' outbreak occurred in March, 2020. As a consequence, there was a lack of revenue not only for cruise lines, but also for cities, ports, and employees. The huge consequences have been impacting in different manners all of the stakeholders, and also customers who used to choose this kind of trip as their main leisure.

Trying to resume as soon as possible, in December 2020, there were 18 cruise lines operating, but the second wave of COVID-19 stopped them again. Nowadays, there are many cruise lines that are going to resume until the end of 2021. In order to make this plan a reality, there are many health and safety protocols, which can vary if the cruise requires or not the mandatory vaccine.

The main protocols include screening travelers' temperature, filling out a health questionnaire and a medical review, taking the COVID-19 Swab Test (some cruise lines are requiring also for vaccinated guests), wearing a mask, keeping social distance, requiring hand hygiene all the time. Dining in buffet only with crew assistance and reduced number of tables for no vaccinated cruises. Splitting guests in all places, such as theater shows, sports and entertainment activities. Going ashore only in a bubble cruise lines excursion.

Table 1 shows the main differences between only vaccinated guest cruises and regular cruises that do not require the mandatory vaccine.

During the COVID-19 pandemic, there are some research works that study the consequences of customers' behavior and preferences in taking a cruise again. Holland [7] in their article named "Cruising through a pandemic: The impact of 
Table 1. Comparation between cruises for vaccinated guest and no mandatory vaccine.

\begin{tabular}{ll}
\hline Cruises with & Cruises with \\
mandatory vaccine & not mandatory vaccine
\end{tabular}

Mask is not mandatory for crew and guests in outdoor and indoor areas. In the port it is mandatory to wear masks.

No need for social distancing.

Boarding allowed even with symptoms.

Testing is not required on embarkation and disembarkation.

Meals with no social distancing requirements and buffet is also possible.

Going ashore is possible without booking cruise lines excursions. Wearing Mask depends on local legislation.

Examples: Oceania, Regent and Celebrity.
Mandatory mask, with rare exceptions, in indoor areas. External areas depend on each company.

There may be designated areas for vaccinated people only. In regular areas, social distancing is requested.

Boarding not allowed if there are symptoms. Prior contact and without symptoms are at the discretion of cruise lines.

Testing before and on landing. Testing during the trip depending on the duration of the trip.

Distance is required, buffet is eliminated. Released the absence of a mask just for eating and drinking.

Going ashore is possible only if booking cruise lines excursions. Wearing Mask is mandatory without being fully vaccinated.

Examples: MSC e COSTA (most popular in Brazilian cost).

COVID-19 on intentions to cruise" investigated the impact of covid-19 on willingness to cruise between cruisers and non-cruisers who live in Australia and the United Kingdom. One of their conclusions is that the country of residence is what influences the most, not only the risk perception, but also their intention to cruise again.

Another interesting research conducted by Radic et al. [8] investigated the behavioral intentions among female passengers for dining on a cruise ship during the COVID-19 era. They also investigated the influence of dining experience scape in the female choices and risk perceptions. Yonggang Lu \& Qiujie Zheng [9] presented a paper about emotional involvement from cruisers according to their activities in the Twitter social media from 1 February until 18 June 2020.

The Cruise Critic [10], an international and huge cruise site has conducted a quantitative survey in order to know the customers' intentions to cruise again. Its survey involved more than 2500 clients of different parts of the world during the year 2020 [10]. According to this research, $83 \%$ of customers will book a future cruise, while $67 \%$ of those are confident about cruise lines' safety procedures.

Although these research papers have brought up a customers' good intention about going back on a cruise during this pandemic situation, there are no data from cruisers' preferences and fears facing the new protocols on board and on shore. Attempting to address this issue, this research paper aims to capture these feelings from a Brazilian perspective.

There are two research questions in this paper. The first one is to identify if the health and safety protocols have an influence on the guests' perception about cruise experience during and post COVID-19 and which protocols influence the most their perception about leisure and safety. The second question is to esti- 
mate how many guests are expected in the next Brazilian cruise season 21/22. The lack of information about the reaction of the cruisers concerning their willingness to go on a cruise the moment they become available was the main motivation to carry on a quantitative survey. The findings of the survey are rather important not only for the planning of the forthcoming season in Brazil, but also for the information necessary to feed the point prediction model presented in the second part of this paper. Important to mention that this survey is unique, in the sense that ever since March 2020 when the 19/20 cruising season had to be interrupted due to the spread of the COVID-19 epidemic exploded. The purpose of the paper is, therefore, twofold; find out the willingness of cruisers after the probable resume of the cruise industry in Brazil and, get the information necessary to create scenarios for the prediction of the number of cruisers for the forthcoming industry.

\section{Survey Methodology}

The information gathered from the review of surveys conducted in other countries related before, and the discussions among the members of the research group created to study the aspects related to the cruise industry in Brazil were used to develop an instrument of data collection to be used in a quantitative survey described in what follows. The instrument of data collection to be used in any quantitative survey constitutes the fundamental tool for the success of the survey. Usually, a qualitative kind of survey is used in order to obtain the important points to be implemented in the instrument. In the present case, the instrument was a consequence of both, previous surveys carried out abroad and the deep discussions among the research group created by the authors of this article.

The quantitative survey was conducted using the SurveyMonkey platform and the Data collection period was April 13th to May 13th, 2021. The survey universe target was of legal age, preferably those who have already been on a cruise trip (at least $70 \%$ of the sample). The means of dissemination was cruise ship groups on Facebook, people triggered by WhatsApp and email.

The survey instrument had three parts. In the first part, there were some question to qualify the respondent, regarding their age and numbers of cruises already taken. Subsequently, the second part addressed the respondents' predisposition to travel, with immediate travel options being given (as soon as there is the possibility of joining a cruise), travel after the end of the immunization process against COVID-19, travel after the complete end of the pandemic and the option to never travel on cruise ships again.

Continuing, the instrument presented the main sanitary procedures that are in force on cruises that are taking place in this return phase while the pandemic is in effect. For the answers, an example of what has already happened with the respondent's predisposition to travel, only three options were used, being "strongly agree", "strongly disagree" and "neither agree nor disagree". 
The new protocols presented in the questionnaire included the need to fill out forms, perform tests, need frequent hand hygiene, social distancing and use of masks, changes in the buffet system, onboard entertainment, going onshore only on cruise line excursions, and the existence of contact tracking among people during the trip.

In addition, the survey instrument also addressed the respondent's perception of the benefits, at this time of the pandemic, of traveling on smaller ships, with fewer ports of call during the trip, having only passengers of the same nationality and shorter trips or nowhere cruises. Other additional aspects were also investigated, such as: the respondents' fear to travel on ships that have had COVID-19 cases on board, the current book for new trips for the 2021/2022 cruise season, and whether travel on cruise ships is their preferred type of trip.

Trying to crossover some data from the sample, in addition to the variable age and number of cruises, the monitoring of news about the pandemic on board cruises was also investigated, as well as the possession of future cruise credits due to canceled trips.

Finally, based on historical time series of the previous cruising seasons in Brazil, as well as on the findings of the survey, it was set a point prediction for the expected number of cruisers for the forthcoming Brazilian 21/22 season. It was adopted a kind of Bayesian argument considering as prior information the official offered of beds for this season and the posterior estimation generated for three possible scenarios.

\section{Survey Results}

As mentioned before, the instrument used to collect the responses was implemented in the Survey Monkey platform that is a powerful and reliable tool that allows all sort of control of the responses. Also, it provides on-line information of the number of respondents and the corresponding characteristics in order to avoid possible bias. That was the case of the sample balance, it was set from the launching of the survey that the sample should have a minimum percentage of respondents that had already been in a cruise (70\% in this survey) against a lower percentage (up to $30 \%$ ) of respondents that had never at all been in a cruise. The dynamic way the Survey Monkey operates allows this sample balance.

As a result, this survey reached 412 responses in a month of application, which implies in the maximum error of $5 \%$ in the estimation of population quantities with $95 \%$ confidence interval. Regarding the age group of respondents, $8.3 \%$ are up to 30 years old, $29.4 \%$ are between 31 and 45 years old, $29.7 \%$ are between 46 and 60 years old, while $32.6 \%$ of the sample are aged over 61 years old. What evidences a good age distribution of the researched sample.

Another important aspect used to classify the sample was the number of cruises previously performed. Among the respondents, $26.2 \%$ had never taken any cruises, $43.2 \%$ had taken 1 to 3 cruises, $13.4 \%$ had taken 4 to 6 cruises and $17.2 \%$ had taken more than 7 cruises. This cruise experience rating will be one of the cross- 
overs shown in this section.

Figure 1 highlights that a value considered from the sample (84.2\%) does not intend to eliminate cruises as a travel option, despite of the dangers associated with the COVID-19. However, 66.5\% stated that they will only travel by ship after complete vaccine immunization. Half of the surveyed sample declares being able to travel as soon as this possibility becomes a reality.

The same answers are shown by crossovers with the number of previous cruises done and having or not previous credits due to cancelled cruises.

Figure 2 shows that the percentage of people who want to go on a cruise as soon as possible was influenced by the number of previous cruises the person has done before. As much experience the people have, the more flexible they are. As shown in Figure 3 there is the same correlation when the disinterest to go on cruise again was investigated.

All people in the sample who have been on a cruise many times (more than 4

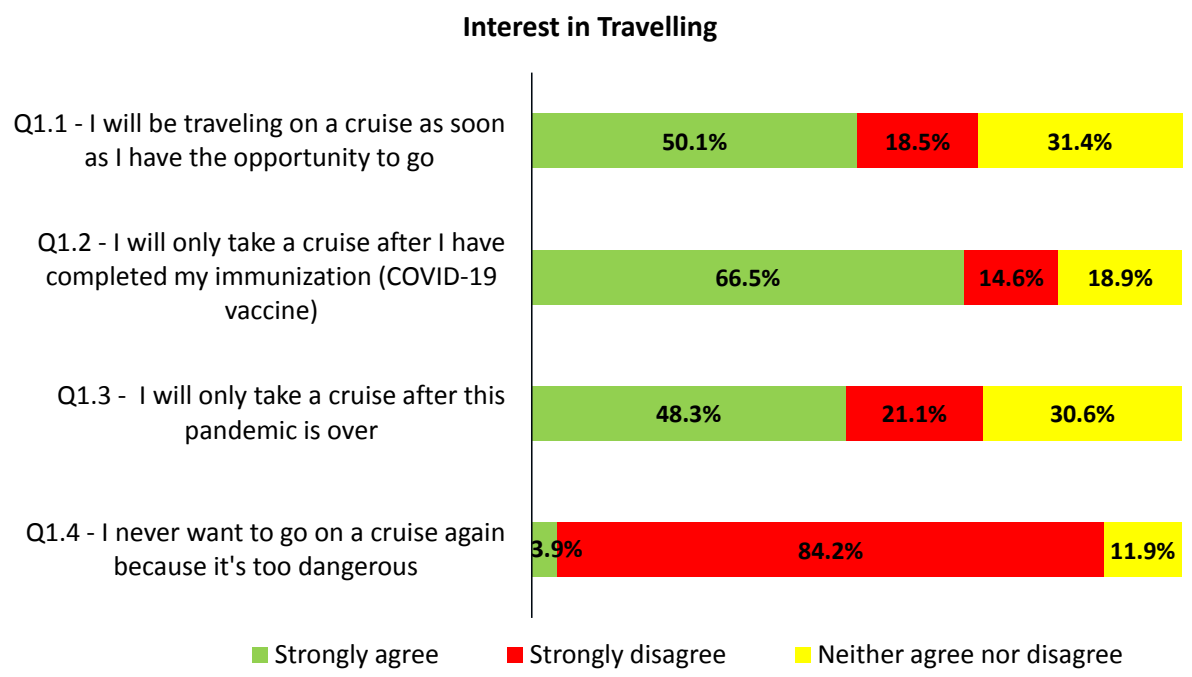

Figure 1. Interest in travelling after the COVID-19.

Q1.1 - I will be traveling on a cruise as soon as I have the opportunity to go

Q3: I've never been on a cruise

Q3: 1 to 3 cruises

Q3: 4 to 6 cruises

Q3: Over 7 cruises

Strongly agree

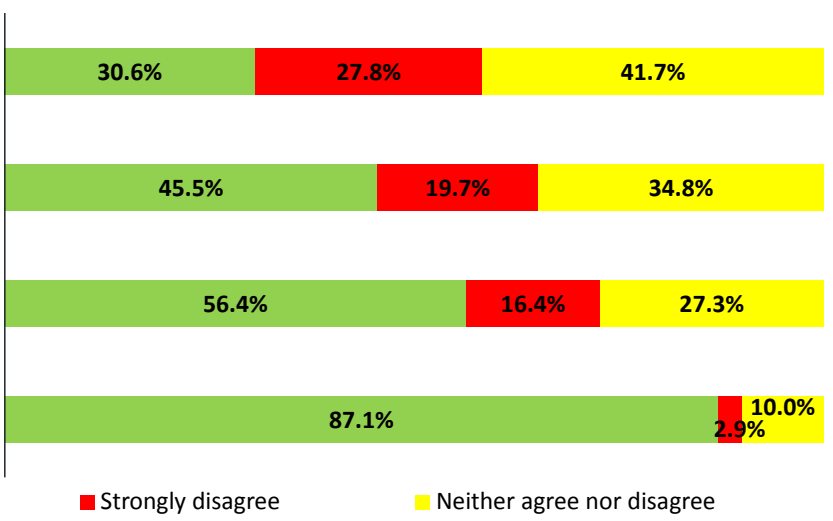

Figure 2. Interest in travelling after the COVID-19 crossover with the number of previous cruises done. 


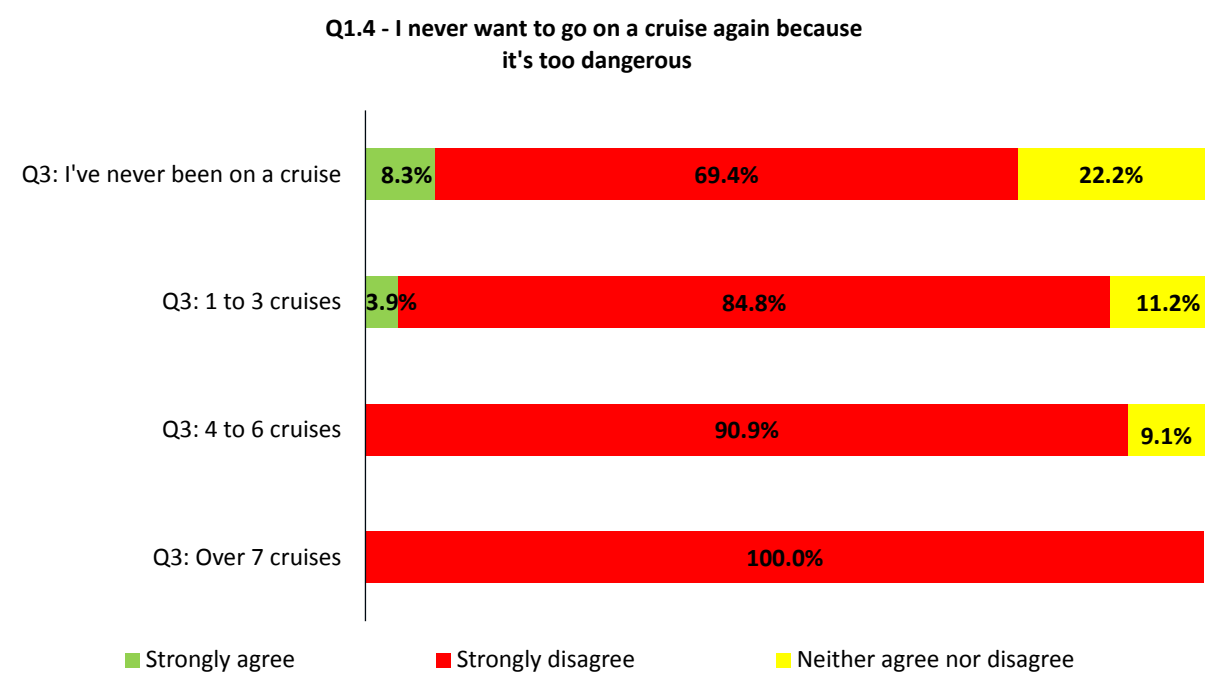

Figure 3. Disinterest in going on a cruise crossover with the number of previous cruises done.

time) are not disinterested to go on a cruise again. Another relevant aspect that influence the desire of going on a cruise is having credit due to cancelled trips, as showed in the Figure 4.

Figure 5 shows the results of the sample's perception regarding filling new forms, taking COVID tests, keeping distance, wearing mask and enjoying buffet with assistance.

Among the findings in these questions, it is observed that most respondents (above 62\%) declare that they do not mind taking the tests, filling out health documents, wearing a mask, maintaining a social distance and using the buffet in an assisted manner by the crew. On the other hand, 33.1\% believe that distancing changes in the experience in leisure and entertainment activities.

Figure 6 deals with sample's perceptions about the assisted excursions, use of contact trackers and reduced occupancy on ships.

Cruises with reduced capacity and the use of wristbands that allow reducing contacts among people and tracing then were not identified as problems by most respondents. In contrast, the possibility of leaving the ship only if booking excursions from cruise lines was reported as a problem for $36.3 \%$ of respondents, while $19.5 \%$ did not have an opinion formed during the response to this survey. Figure 7 addresses aspects related to trip duration and the number of ports of call.

The results presented in Figure 7 demonstrate that most respondents do not prefer shorter cruises or stops in fewer number of ports at this stage of the pandemic, in the other words, the cruise size and number of ports do not represent, for most respondents, aspects that add security in this resumption of cruises. This data was also crossover with the quantity of previous cruises done by respondents and the preference for shorter cruises or fewer number of ports decrease while the number of cruises done increase. These results are showed in Figure 8 and Figure 9. 
Q1.1 - I will be traveling on a cruise as soon as I have the opportunity to go

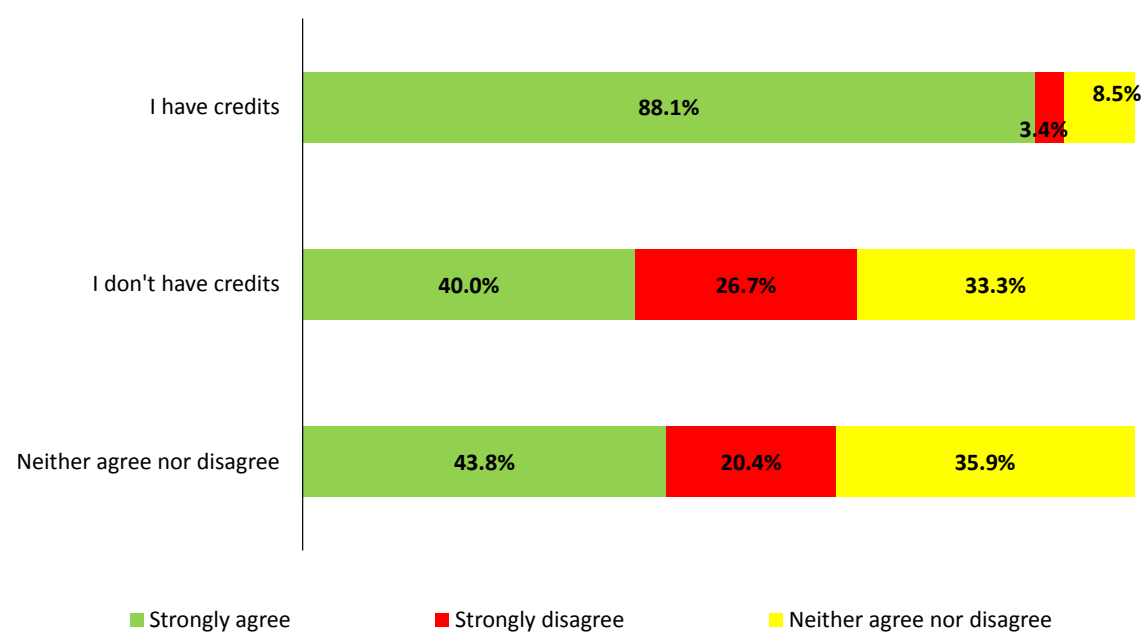

Figure 4. Interest in going on a cruise crossover with having previous credit with cruise companies.

Forms, Distances and Buffet

Q1.5 - I don't mind filling out the health forms, taking COVID tests before, during or after the cruise, wearing a mask on board, always sanitizing my hands and maintaining social distance on board

Q1.6 - Social distancing in leisure and entertainment activities does not change my pleasure of being on board

Q1.7 - The buffet service served by the crew does not interfere with my experience on board

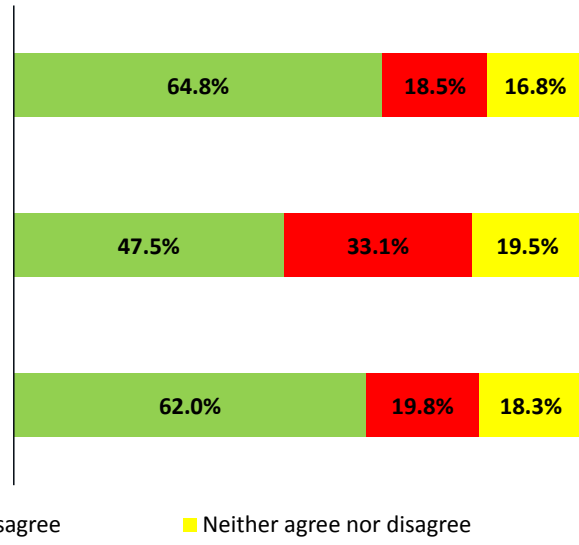

Strongly agree $\quad$ Strongly disagree $\quad$ Neither agree nor disagree

Figure 5. The sample's perception regarding forms, tests, distances, and buffets.

\section{Tours, Bracelet and Reduced Occupancy}

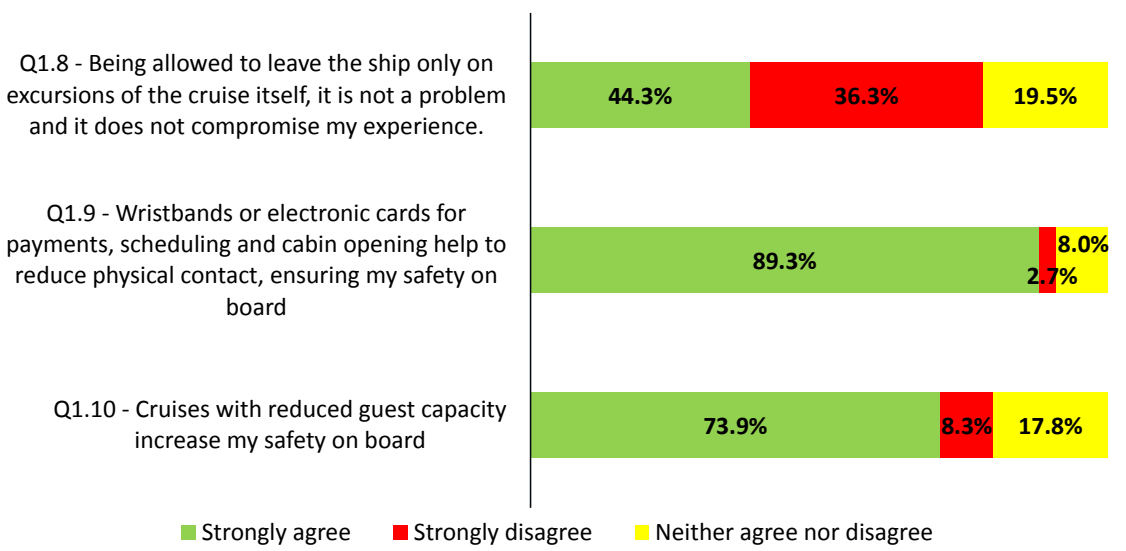

Figure 6. The sample's perception regarding excursions, wristbands, and reduced capacity. 
Trip Duration and Stopover Ports

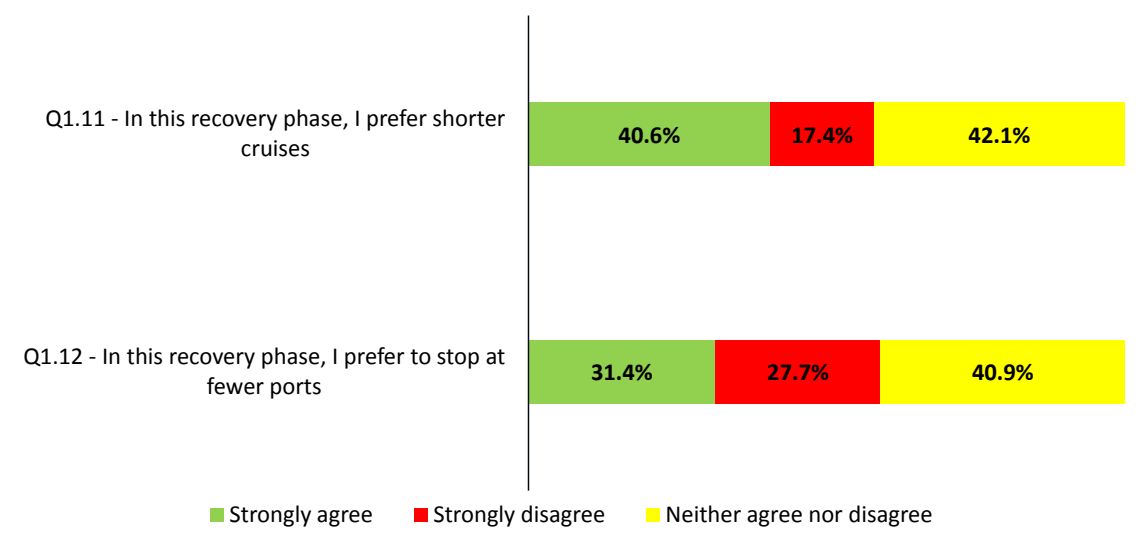

Figure 7. The sample's perception regarding to the trip duration and ports of call.

Q1.11 - In this recovery phase, I prefer shorter cruises

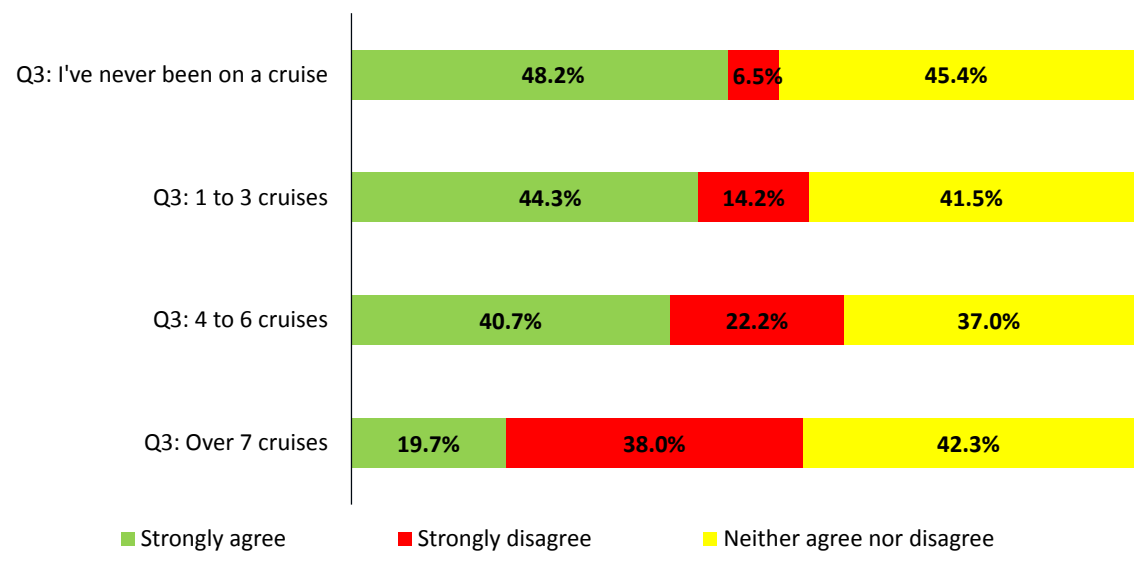

Figure 8. Preference for shorter cruises crossover with the number of previous cruises done.

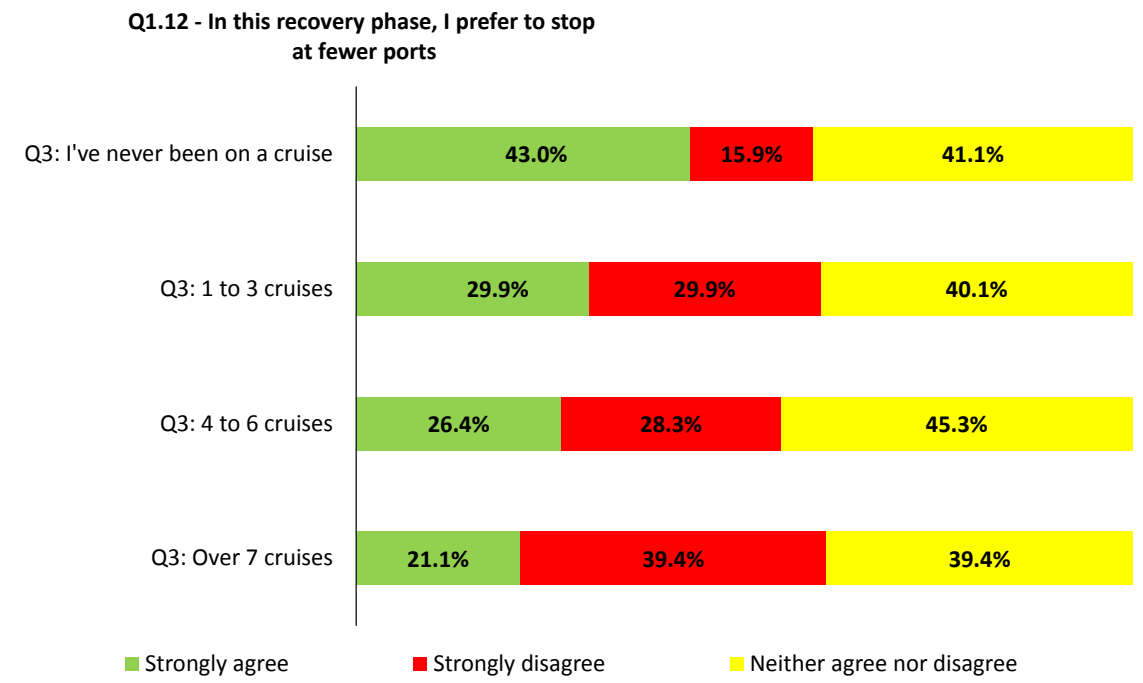

Figure 9. Preference for fewer ports of call crossover with the number of previous cruises done. 
Figure 10 shows the influence of the absence of stops during cruises, regarding the nationalities of passengers on board and the size of the ship on the safety perceived by the respondents.

The three aspects showed in Figure 10 do not represent security in the perception of respondents to this survey, that is, the majority (over 50\%) disagrees that enjoying a nowhere cruise, or with passengers of a single nationality, or traveling on smaller ships to increase their safety on board during travel in the pandemic.

Figure 11 shows the characteristics of the sample regarding the monitoring of news about COVID on board ships and the fear of traveling on a ship where there were infected people on board.

Figure 11 shows that the majority of people have (64.9\%) followed news about the pandemic on board cruise ships, while few respondents (30.95) declare that they are not afraid to travel on a ship that has already been infected with cases of COVID-19.

Figure 12 presents the quantity of people who have Future Cruise Credit (FCC), scheduled trips for the next seasons and their travel preferences.

The sample of this research was characterized by having people with paid and canceled travel credits (only 14.7\%), while a very similar proportion $(17.3 \%$ ) were purchasing trips for the 2021/2022 season. Regarding the declaration of preference for cruises as the best travel option, this was identified in $30.6 \%$ of the sample.

This data was also crossover with the quantity of previous cruises done by respondents and the number of people who have FCC and booking new cruises increases while increases the number of cruises done. These results are showed in Figure 13 and Figure 14.

The same effect is perceived from people who affirm cruise trip is the best kind of trip for them, as it is presented in Figure 15.

Absence of Stops, Nationalities on Board and Ship Size

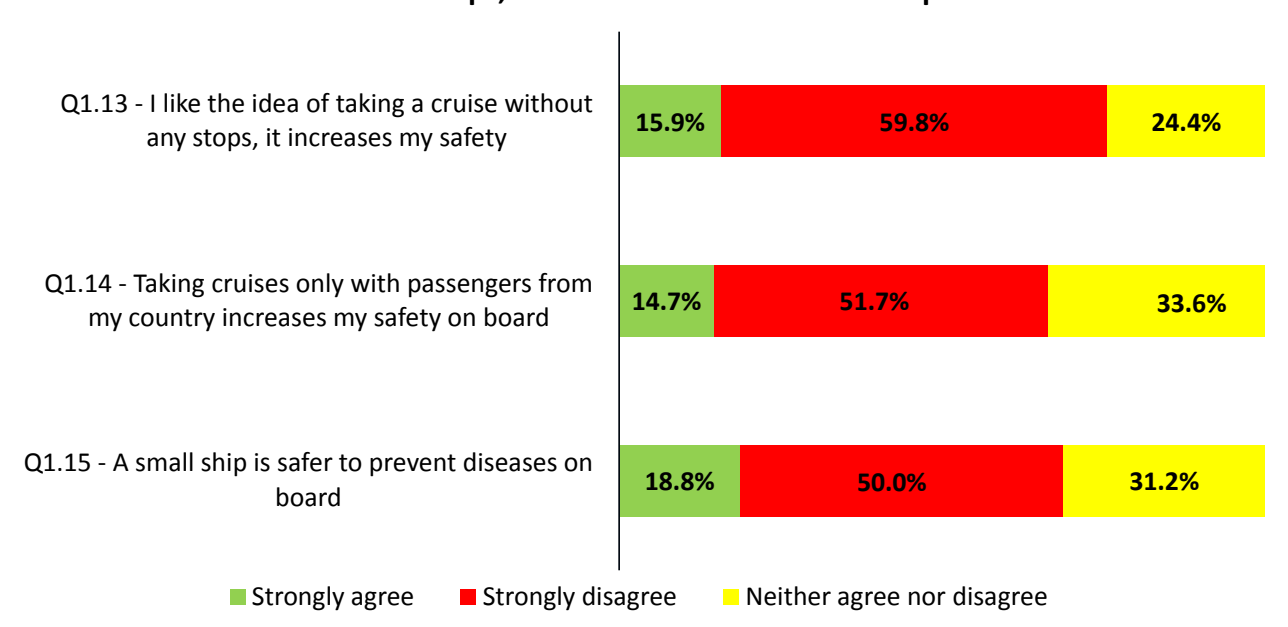

Figure 10. Preference for nowhere cruises, nationalities on board and ship size. 


\section{Media and Fear of Traveling}

1.16 - I followed the news about epidemics aboard cruise ships in the first quarter of 2020

$64.9 \%$

$16.6 \%$

Q1.17 - I have no fear of traveling on a ship that has been infected and there have been deaths on board

Neither agree nor disagree

Figure 11. Monitoring the news about COVID in cruise ships and fearing of going on a cruise again.

\section{Credits, Scheduled Trips and Preferences}

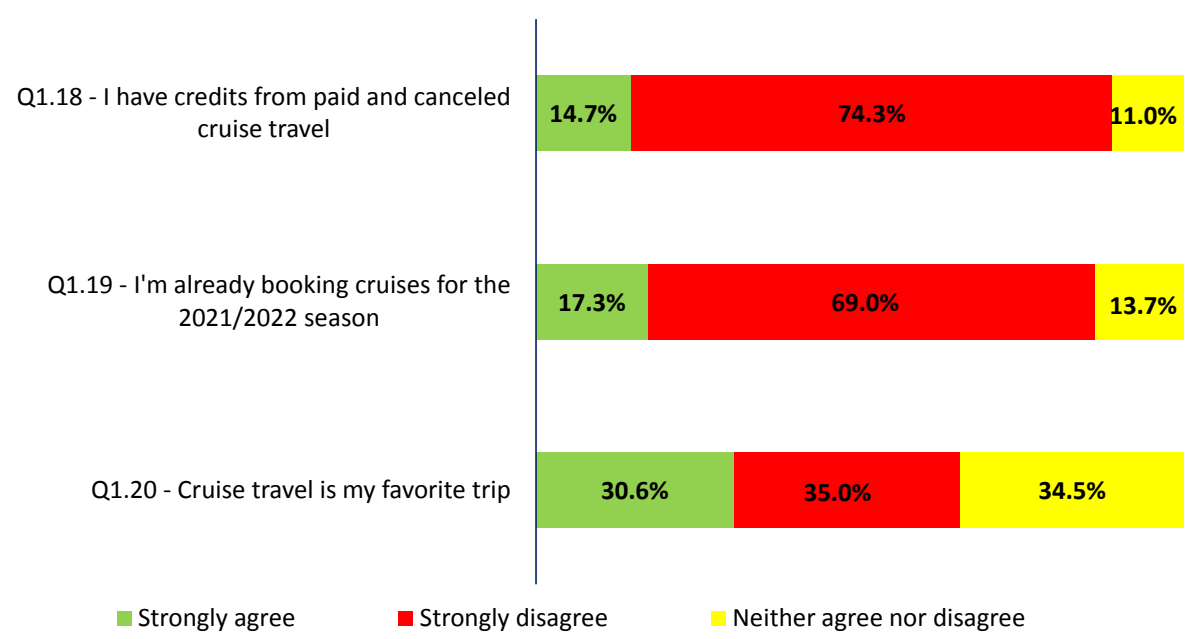

Figure 12. Future cruise credits, scheduled trips and travel preference.

Q1.18 - I have credits from paid and canceled cruise travel

Q3: I've never been on a cruise

$$
\text { Q3: } 1 \text { to } 3 \text { cruises }
$$

Q3: 4 to 6 cruises

Q3: Over 7 cruises

Strongly agree

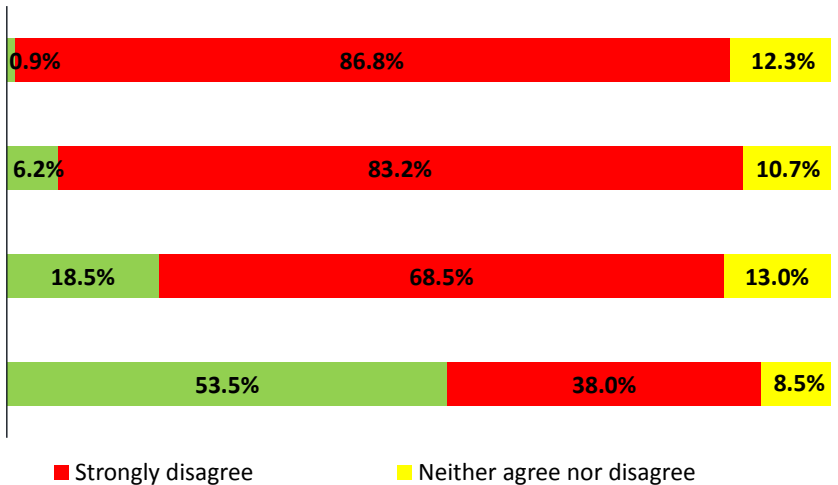

Figure 13. Future cruise credits and number of cruises done. 
Q1.19 - I'm already booking cruises for the 2021/2022 season

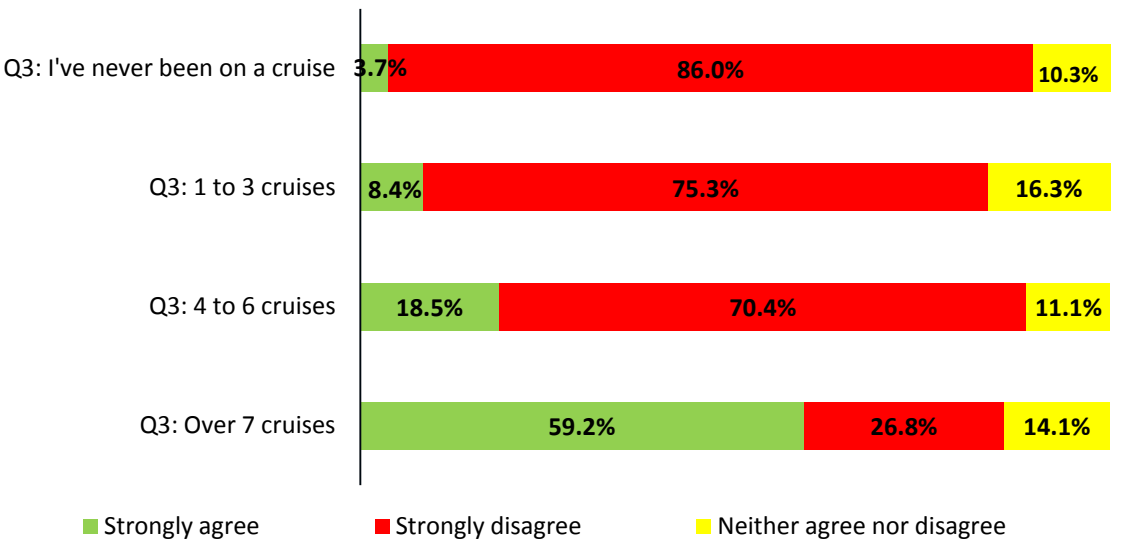

Figure 14. Booking future cruises and number of cruises done.

Q1.20 - Cruise Travel is my Favorite Trip Q3: I've never been on a
cruise

Q3: 1 to 3 cruises

Q3: 4 to 6 cruises

Q3: Over 7 cruises

Strongly agree
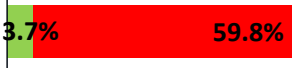

$36.5 \%$
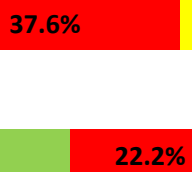

$88.6 \%$

Neither agree nor disagree

Figure 15. Preference for cruise trip and number of cruises done.

\section{Survey Discussion}

This research points some pros for resuming the cruise industry in Brazil. The majority of the samples don't mind to obey the majority of new health and safety protocols. This flexibility increases in the same way people have gone more time on a cruise. Another important result is that most of people (66.5\%) are going on a cruise as soon as being fully vaccinated, while only $3.9 \%$ will not go on a cruise due to fear of COVID-19.

Some new protocols are bringing confidence for people about feeling more secure onboard. For $74 \%$ of sample, the lower capacity is recognized as an improvement of the health conditions, while $89.3 \%$ of respondents affirm the same for easy contactless services.

The most important core issue for the cruise industry is the high Brazilian's rejection for going onshore only if booking "bubble excursions" from cruise lines. 
There are many reasons for this perception. First, cruise lines excursions used to be much more expensive than hang out with friends or family by their own. Second, the cruises will be only in Brazilian coast or in neighbor countries in the South America, as a consequence, the idiom is not a problem for the guests, which could incentivize not to be on their own.

Another issue is the requirement to keep social distance all the time. It has huge influence in customer's experience. Brazilians have special preference for thematic cruises, including singers and shows, and it looks very difficult to keep social distance in these kinds of parties.

The majority of respondents (65\%) have followed the news about COVID-19 on cruise ships. As a consequence, almost $48 \%$ of sample answered that will mind going on cruise in a previously infected ship. Additionally, there were $21.4 \%$ of respondents that did not know yet their perception about this issue. This group could be better prepared to accept or not go on a previously infected ship without matter about it.

It is incredible how the perception could differ from the reality in the costumer mind. Small ships, reduced ports of call or nowhere cruises, and a single nationality on board are not perceived as a safety cruise for the respondents. Despite the fact that there is no scientific research that affirms the main cause to spread COVID-19 on board, it is possible to infer that less people, ports and different nationalities on board could represent more security than the opposite situation.

During the data collection, only $17 \%$ of samples are booking cruises for the next season, while $15 \%$ of samples have future cruise credit (FCC) from previous cancellation. The similarity between these answers is not a coincidence, probably people who are booking during the COVID-19 are the same that have FCC and must book until some specific date.

Using these survey results is possible to predict that as soon as COVID-19 will be on control and the population will be fully vaccinated, there will be a boom of booking cruises in Brazil.

\section{A Bayesian Point Prediction of the Number of Cruisers in Brazil Season 21/22}

The classical Bayesian parametric approach to predict the future values of a time series variable requires the knowledge of its conditional parametric distribution for this time series stochastic process. The corresponding unknown parameters of such distribution is also a stochastic process and its time evolution is described by probability distributions that are sequentially updated as the time series observations are observed. Such updating procedure constitutes the prior/posterior scheme that characterizes the Bayesian inference procedure. In the case of time series prediction, the predictive distribution is sequentially obtained by the convolution of both; the parametric time series distribution and the posterior parameter distribution generated sequentially as the time evolves [11]. The classi- 
cal Bayesian inference as briefly presented is entirely described in terms of probability distributions, due to the fact that the time series and the corresponding parameters are treated as random variables and/or stochastic process. As such, the modelling requires their respective probability distribution. The Bayesian principle is related to the sequential updating procedure to the parameter distribution (prior/posterior scheme) and the time series predictive distribution.

In the present study, it is only considered the idea of inferring the point prediction of the total number of cruisers for the forthcoming cruising industry in Brazil. Although this number is unknown and, therefore, using the Bayesian principle, should be treated as a random variable, it is carried out a point prediction for Nt (number of expected cruisers), without any assumption of probability distribution for $\mathrm{Nt}$, nor any parameter associated to it. It is only considered a prior/posterior point prediction described applied to $\mathrm{Nt}$ in a deterministic way. The prior for Nt is the official number of beds published by the cruise industry for the 21/22 Brazilian season, while the posterior point prediction for $\mathrm{Nt}$ is given by a set of possible scenarios generated from side information and the findings of the survey.

As mentioned before, in this final section it will be presented an estimation of the expected number of cruisers for the 21/22 cruising season in Brazil. For that, a kind of Bayesian argument [12] [13] is used, where, based on the historical number of cruisers in Brazil ranging from 04/05 up to 19/20 seasons. Important mentioning that the figures for the 20/21 season are not included as all the cruisers for this season were cancelled due to the COVID epidemic. In Table 2 are shown the figures of the last 15 seasons including the number of ships (row 1); number of cruisers (row 2) and the yearly growth rate (row 3). These numbers of cruisers are also plotted in Figure 16.

In order to set a deterministic prior for the number of cruisers expected for the 21/21 season in Brazil, some important findings of the survey described in the previous sections were considered:

1) Approximately $67 \%$ of the interviewed consider going on cruise holidays after being completely immunized;

2) The majority of the respondents that has already have an experience of at least one cruise, considers taking a cruise holidays after being completely immunized. This is also true whatever the age group of the respondents is;

3) No negative effect of the covid-19 on future choice of cruise holidays once the pandemic is over.

Also, some other important information collected from the available historical data were also considered in setting the prior estimation of the number of cruisers for this upcoming season. It was considered the number of beds available for the 19/20 season and the corresponding occupancy of beds in this season; i.e.:

Offer of beds 19/20: 530,000;

Number of cruisers 19/20: 469,577; 


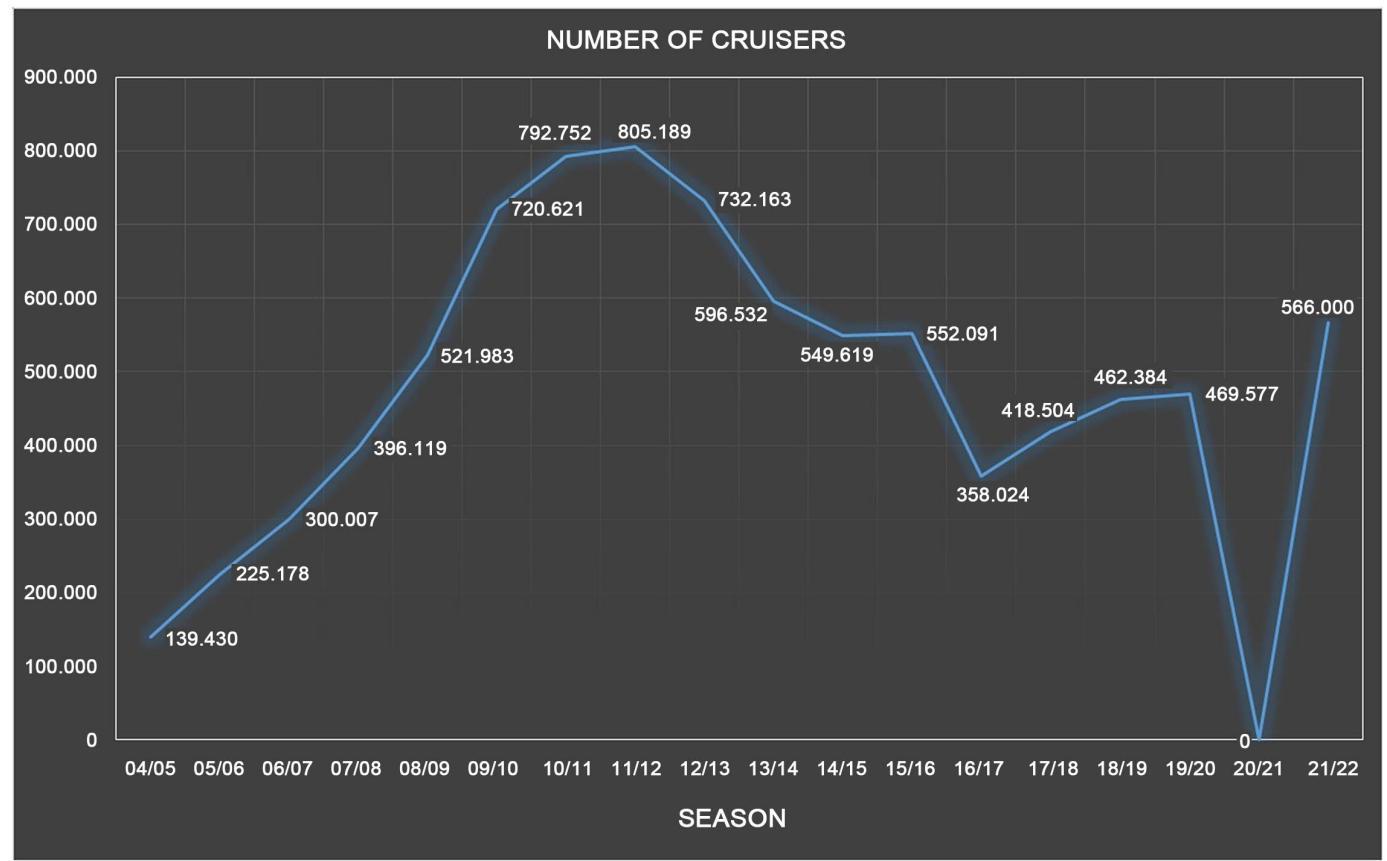

Figure 16. Plot of the historical number of cruisers in Brazil.

Table 2. Historical data of cruisers in Brazil from seasons 04/05 up to 19/20.

\begin{tabular}{ccccccccc}
\hline Season & $13 / 14$ & $14 / 15$ & $15 / 16$ & $16 / 17$ & $17 / 18$ & $18 / 19$ & $19 / 20$ & $20 / 21$ \\
\hline $\begin{array}{c}\text { Number of } \\
\text { ships }\end{array}$ & 11 & 10 & 10 & 7 & 7 & 7 & 8 & 8 \\
$\begin{array}{c}\text { Number of } \\
\text { cruisers }\end{array}$ & 596.53 & 549.61 & 552.09 & 358.02 & 418.50 & 462.38 & 469.57 & $N / A$ \\
Growth rate & $-18.50 \%$ & $-7.90 \%$ & $0.40 \%$ & $-35.2 \%$ & $11.7 \%$ & $10.5 \%$ & $1.50 \%$ & \\
\hline
\end{tabular}

Rate of occupancy 19/20 season: $~ 88.5 \%$;

Official offer of beds for the 21/22: 566,000.

This can be considered the as the prior expectation of the number of cruisers for this forthcoming season. The rationale behind this choice is the fact that the cruiser lovers would be very much interested in jumping into a cruise the moment they are available after almost two years of interruption. Also, important to mention that the 19/20 season was interrupted in mid-March/2020 when the epidemic was official declared by the World Health Organization. There were still some cruisers booked till the mid-April/2020 when the Brazilian season ends. For this reason, it does make sense adopt these 566,000 occupancies as the prior point prediction of cruisers for the $21 / 22$ season. In order to derive point posteriors predictions, it was considered 3 scenarios described below:

Scenario 1: Optimistic Posterior Point Prediction

Assumptions:

Brazilian population above 12 years old fully vaccinated by November/21;

Same occupation rate as 19/20 ( 88.5\%);

Optimistic posterior point prediction: 500,910 cruisers; 
Scenario 2: Conservatives Posterior Point Predictions

Assumptions:

Population above 12 years old fully vaccinated by November/21;

Same occupancy rate of season 19/20 ( 88.5\%);

Considers only $70 \%$ of the available beds offer; i.e., 396,200 beds;

- (2.1) Only 19/20 occupation rate (88.5\%).

$>$ Conservative posterior point prediction (1): 350,637 cruisers

- (2.2) Same 19/20 growth rate; $1.50 \%$

$>$ Conservative posterior point prediction (2): 355,897 cruisers

Scenario 3: Realistic Posterior Point Predictions

Assuming maximum ship occupancy rate of $70 \%$ due to:

ANVISA (Brazilian equivalent to CDC) has not yet allowed the arrival of the 7 ships scheduled for 21/22 season in South America;

Vaccination rate in Brazil still low. Figures of 25th June 2021: 33\% of the total population vaccinated with first dose (13\% with second dose);

On the other hand, due to recent reviews of the vaccination schedule in the main ports in Brazil (São Paulo \& Rio de Janeiro), it is expected that by December/21 the whole population above 12 years of age will have received the first dose;

$\checkmark$ The characteristics of the ships scheduled to cover 21/22 season in Brazil are somehow preferable for family cruisers. This could imply in a reduction of cruisers holydays for Brazilian families that may not feel secure to take their non-vaccinated children in a cruise.

Also, in order to obtain the posterior point predictions for this realistic posterior point prediction, the following further assumption was considered; maximum ship occupancy rate of $70 \%$ due to:

Although the survey reveals that approximately $67 \%$ of the respondents are willing for a cruise holiday once they are fully vaccinated; we have to consider the possibility of a reduction of this preference if the children are not vaccinated until the December/21.

Based on the above considerations, the following assumptions will be set in order to arrive at realistic posterior point projections:

1) $70 \%$ maximum occupancy restriction (offer of 396,200 beds);

2) $67 \%$ willing for a cruise holiday once immunized (prediction 3.1 );

3) Reduction of $20 \%$ due to restriction of children on board (prediction 3.2).

(3.1) Realistic Posterior point prediction (1): 234,927

(3.2) Realistic Posterior point projection (2): 187,942

Finally, these prior/posterior point predictions are summarized below:

Prior: 566,000 cruisers;

Posterior optimistic: 500,910 cruisers.

Posterior Conservative:

1) Without growth: 350,637 cruisers;

2) $19 / 20$ growth rate: 355,897 cruisers. 
Posterior Realistic: 500,910 Cruisers

1) Limited occupancy: 234,927 cruisers;

2) Children constraint: 187,942 cruisers.

Posterior Pessimistic: No Cruisers at all

Concerning possible evaluation of these point predictions, one has to wait the outcome of the season (ending in April/22) to learn which of the scenarios best describe the occupancy rate of beds for the Brazilian 21/22 season. By the time this paper was written, the posterior pessimist scenario could be almost disregard as the number of registered COVID cases in the country started a decreasing trend, the vaccinations all over the country speed up and the travel agencies were offering and selling cruise holidays betting the season would happen for sure.

\section{Conclusions}

The international cruise industry was paralyzed in April 2020, when the COVID19 international pandemic was decreed by the WHO. Since then, the industry has kept its fleet of ships at a standstill with only a minimal crew to ensure their safety. This significantly impacted its entire production chain, which includes, in addition to the crew, the entire marketing, operation, supply chain, as well as impacts on ports of call and embarkation and disembarkation cities, where in many cases there was an important economic reduction. The industry suffered significant losses resulting from the absence of operations, which led, in some cases, to liquidation, bankruptcy and sending several vessels to premature cutoff [14].

The resumption of the cruise industry is not yet the full reality, although some experiences have taken place at the end of 2020, it was again paralyzed due to the second wave associated with COVID-19. Parallel to this, advances have occurred after the beginning of COVID-19 vaccination in the world. This evolution in the mass vaccination program in some strategic regions and countries for the cruise industry, such as the USA and Europe, brings hope that a partial, albeit more significant recovery has started in July 2021.

Safe resumption will only be possible if cruise lines adopt a new onboard routine, which differs for cruises that only allow the vaccinated population ( $95 \%$ of guests and crew) onboard or those that do not require vaccination and adopt extensive testing of the entire public.

Aiming to identify a potential Brazilian guest's perception of these new protocols, associated with the secondary objective of identifying their predisposition to travel on cruises while the pandemic was in force, the quantitative primary research was developed, which was applied during the period from April to May 2021, with 412 respondents.

The sample surveyed can be considered well distributed, both from the point of view of age group, as well as previous experience in going on a cruise. The greater predominance of intention to travel again on cruises was associated with the com- 
pletion of the immunization process against COVID-19. Only $4 \%$ declared that they have no intention of traveling on cruises again. Among the investigated sample, only $30.6 \%$ declared their preference for cruise travel, while $26 \%$ had never traveled on a cruise ship.

Some data crossings were carried out, where it was possible to verify that the age group had little influence on most answers, while the number of cruises carried out influenced most answers, particularly on the group who have taken more than 7 cruises. In this case, a very favorable position is clearly identified, not only for traveling immediately, but also for adapting to the new protocols.

Another factor that greatly influences the willingness to travel immediately is having future cruise credits. This same audience declares that they are already making reservations for the 2021/2022 cruise season. Monitoring the news about the pandemic on cruise ships does not interfere with the responses collected.

Among the new protocols already implemented in cruise ships, the obligation to book cruise line excursions in order to be allowed to go onshore has the highest percentage of rejection. What's surprising the most in the sample's answers is the lack of association with onboard safety for these situations: smaller ships, passengers of the same nationality, shorter trips, fewer ports of call, or cruises for nowhere.

Most respondents do not mind filling in health documents, taking the COVID19 test, making hand hygiene all the time, wearing masks, maintaining social distance and having assistance in buffet services. The sample associate the passenger reduced capacity and the use of tracking devices to reduce physical contact as an increase in the safety of the cruise.

It should also be noted that the 21/22 season in South America is scheduled, with an initial offer of 566,000 beds for the seven ships allocated. However, this Brazilian season is still waiting for approval from responsible agencies, which are observing the evolution of a pandemic in the region. It is possible to predict the high vaccine dependency to the cruise industry resuming in Brazil 21/22.

The aspects raised in this research bring important findings and allow anticipating the perception of Brazilian passengers regarding the new on-board routine scheduled for 2021/2022. This information can contribute to more assertive communication among cruise companies, their marketing partners and the interested public. It also allows for a prediction about the acceptance or not of the new protocols, directing improvements in communication with potential customers.

The number of cruisers point predictions was generated, basically, following previous information of the historical occupancy rate of the Brazilian cruisers and the important findings from the survey conducted by the authors. In fact, by the time these estimates were performed, there was no decision whatsoever from the Brazilian sanitary authorities concerning the possibility of allowing the 21/21 cruiser season to operate. That was the case of the previous 20/21 season. This uncertainty leads to the various scenarios considered in this prediction exercise. 


\section{Conflicts of Interest}

The authors declare no conflicts of interest regarding the publication of this paper.

\section{References}

[1] Pontes, M.C. (2012) Marketing experiencial ou o uso da experiência no marketing? Estudo de caso em hotéis 5 estrelas da cidade de São Paulo. Ph.D. Thesis, Universidade de São Paulo, São Paulo.

https://www.teses.usp.br/teses/disponiveis/12/12139/tde-11062012-200639/publico/ MarceloChiavonePontesVC.pdf

[2] Barríos, M. (2012) Marketing de la Experiencia: Principales conceptos y características. Palermo Business Review, 7, 67-89.

[3] Larocca, M.T.G., Ladeira, R., Da Silva, A.L.L. and Mello, R.C. (2020) Marketing de Experiência: Um estudo dos aspectos conceituais. Cadernos EBAPE.BR., 18, Edição Especial. https://doi.org/10.1590/1679-395120190079

[4] Cruise Lines International Association (CLIA) (2020) State of Cruise Industry Outlook. https://cruising.org/-/media/research-updates/research/state-of-the-cruise-industry. $\underline{\operatorname{ashx}}$

[5] Perucic, D. (2020). Analysis of the World Cruise Industry. In: DIEM: Dubrovnik International Economic Meeting, 5, 89-100.

[6] David, A.B.M. (2016) The Cruise Industry: Past, Present and Future. Journal of Tourism Research, 14, 143-155.

[7] Holland, J., Mazzarol, T., Soutar, G.N., Tapsall, S. and Elliott, W.A. (2021) Cruising through a Pandemic: The Impact of COVID-19 on Intentions to Cruise. Transportation Research Interdisciplinary Perspectives, 9, Article No. 100328. https://doi.org/10.1016/j.trip.2021.100328

[8] Radic, A., Lück, M., Al-Ansi, A., Chua, B.L., Seeler, S., Raposo, A., Han, H., et al. (2021) To Dine, or Not to Dine on a Cruise Ship in the Time of the COVID-19 Pandemic: The Tripartite Approach towards an Understanding of Behavioral Intentions among Female Passengers. Sustainability, 13, 2516. https://doi.org/10.3390/su13052516

[9] Lu, Y.G. and Zheng, Q.J. (2021) Twitter Public Sentiment Dynamics on Cruise Tourism during the COVID-19 Pandemic. Current Issues in Tourism, 24, 892-898. https://doi.org/10.1080/13683500.2020.1843607

[10] Coulter, A. and Faust, C.G. (Eds.) (2021) Cruise 2021: Where the Cruise Industry Stands after a Year without Cruising. https://www.cruisecritic.co.uk/articles.cfm?ID=5901\#peoplestillwanttocruise

[11] Souza, R.C. (1980) A Bayesian Entropy Approach to Forecast. Ph.D. Thesis, Warwick University, UK.

[12] Andrews, M. and Bagule, Y.T. (2013) Prior Approval; The Growth of Bayesian Methods in Psychology. British Journal of Mathematical and Statistical Psychology, 66, 1-7. https://doi.org/10.1111/bmsp.12004

[13] O’Hagan, A. (1998) Eliciting Expert Beliefs in Substantial Practical Applications. Journal of the Royal Statistical Society Series D. The Statistician, 47, 21-35. https://doi.org/10.1111/1467-9884.00114

[14] Silva, A.L.R. (2021) An Overview of the Impact of COVID-19 on the Cruise Industry with Considerations for Florida. Transportation Research Interdisciplinary Perspectives, 10, Article No. 100391. https://doi.org/10.1016/j.trip.2021.100391 